\title{
Alexitimia y funcionalidad familiar en estudiantes de ingeniería
}

Alexithymia and family functionality among engineering students

\author{
Lisha Y. Galagarza Pérez \\ Walter L. Arias Gallegos \\ Universidad Católica San Pablo, Perú \\ Correspondencia: lgalagarza@ucsp.edu.pe
}

Recibido: 05-04-2016

Aceptado: 18-01-2017

\section{Resumen}

La presente investigación pretende valorar la relación entre la alexitimia y la cohesión familiar. Con ese objetivo, se utilizaron como instrumentos dos cuestionarios: FACES III de Cohesión y Adaptabilidad Familiar y la Escala de Alexitimia de Toronto (TAS-20). La muestra estuvo constituida por 423 estudiantes matriculados en el noveno semestre de los cuatro programas profesionales de la Facultad de Ingeniería de una universidad privada de la localidad de Arequipa. Los resultados permitieron concluir que la incidencia de presencia de alexitimia es del $32 \%$ de la muestra evaluada. Además, entre los hombres y mujeres evaluados, el mayor porcentaje de alexitimia lo tienen los hombres, con un $37.50 \%$, frente a un $26.67 \%$ en las mujeres. De las personas con alexitimia, el tipo de cohesión predominante es el tipo semirelacionado ( $45.7 \%$ ), seguido del no relacionado (31.4\%). Mientras que en las personas sin alexitimia, el más predominante es el tipo semirelacionado (42.1\%), seguido del relacionado (22.0\%).

Palabras clave: Alexitimia, dinámica familiar, adaptabilidad familiar, cohesión familiar.

\begin{abstract}
The present research aims to assess the relationship between alexithymia and family cohesion. With this purpose, we used two kind of questionnaires: FACES III Cohesion and Adaptability Scale and Toronto Alexithymia Scale (TAS-2o). The
\end{abstract}


sample was composed of 423 students enrolled in the ninth semester of the four professional programs of the faculty of engineering of a private university in Arequipa. The results of this investigation, allowed us to conclude that the incidence of presence of alexithymia is $32 \%$ of the sample. Moreover, between men and women the highest percent of alexithymia is from men with $37.5 \%$, against $26.67 \%$ of women. The people with alexithymia, has a Semi-related type of Cohesion, followed by Non-related. Students without alexithymia has the Non-related followed by Related type.

Key words: Alexithymia, family dynamics, family cohesion, family adaptability.

\section{Introducción}

La alexitimia fue introducida al mundo académico de la salud mental en 1972 por Sifneos, quien la definió como la dificultad para describir sentimientos, una ausencia o reducción de la fantasía, y la manifestación de pensamiento operatorio (Sifneos, 1972). Desde entonces, se han llevado a cabo diversas investigaciones en las que la alexitimia se ha relacionado con una diversidad de desórdenes mentales, siendo un fuerte predictor de algunos trastornos psicofisiológicos, como el propio Sifneos propuso en 1973 (Sifneos, 1973). De hecho, ya desde 1939, los médicos psicosomáticos, como Flander Dumbar, se referían a ella, aunque no con el nombre de alexitimia.

Hoy en día se ha observado su prevalencia en pacientes psicosomáticos, pero está presente en una gran variedad de desórdenes físicos y psiquiátricos, como la depresión y la esquizofrenia (Alonso-Fernández, 2011). Por ejemplo, se ha reportado su mayor incidencia en pacientes que presentan psoriasis (Torres-Hernández, López-García, Pedroza-Escobar \& Escamilla-Tilch, 2015), trastornos alimentarios (Laquatra \& Clopton, 1994; Taylor, Parker, Bagby \& Bourke, 1996), trastornos gastrointestinales (Diaz, 2011) o en los consumidores de drogas, en quienes hay una prevalencia con valores cuatro veces por encima de quienes no son drogodependientes (García, Rodríguez, Córdova \& Fernández-Cáceres, 2016). Estas relaciones, empero, no siempre se han podido verificar, como en el estudio de Almeida y Machado (2004), donde se concluye que la alexitimia no predice la somatización.

Asimismo, su definición también ha estado sujeta a controversias, pues mientras algunos autores la definen como un tipo de personalidad (Moral de la Rubia \& Ramos-Basurto, 2015), otros la explican como una dificultad para identificar y expresar emociones (Krystal, 1988), como núcleo patognomónico, siguiendo la propuesta original de Sifneos. Sin embargo, se relaciona con una gran variedad de síntomas, por ello, se suele diferenciar entre alexitimia primaria y secundaria (Moral de la Rubia \& Retamales, 2000). 
En el primer caso, los síntomas se mueven en torno a los déficit en el procesamiento emocional y el reconocimiento de estímulos emocionales (Martínez \& Marín, 1997), entre los que se tiene: dificultad para reconocer estímulos vocales emocionales (Martínez, Montero \& Cerra, 2002), dificultad para procesar la expresión de emociones faciales (Donges, Kersting \& Suslow, 2014), escasa empatía (Fernández \& Sánchez, 2002), limitada inteligencia emocional (Mayer, Di Paolo \& Salovey, 1990), estrategias de afrontamiento inadecuados (Roco, Baldi \& Álvarez, 2014), etcétera.

En ese sentido, un mecanismo explicativo de la alexitimia es su relación con el estrés, ya que de ahí pueden devenir una gran diversidad de desórdenes psicológicos y físicos, aunque esta hipótesis no ha sido del todo corroborada a través de estudios empíricos (Martínez, Ortiz-Soria \& Ato-García, 2001). Otras explicaciones tienen que ver con aspectos biológicos, pues se ha encontrado que es más común entre gemelos homocigóticos, lo que relieva las hipótesis genéticas. A nivel cerebral, se ha registrado que en la alexitimia hay una actividad anormal de la amígdala, que regula las emociones (Ledux, 1999), o alteraciones en la corteza prefrontal frontocingular, zona que se encuentra más deteriorada en consumidores de drogas, donde la alexitimia es frecuentemente reportada (Craparo et al., 2016).

La lateralización cerebral también ha servido de hipótesis etiológica, con prevalencia en sujetos que tienen predominio del hemisferio derecho (Alonso-Fernández, 2011). De ahí que la alexitimia sea reportada en mayor medida en el sexo femenino, con una razón de 8 a 1 entre varones y mujeres (García et al., 2016). Aunque, en Arequipa, se realizó un estudio con 566 estudiantes universitarios sobre el reconocimiento de expresiones faciales, donde no se encontraron diferencias entre hombres y mujeres (Morey, 2007). En este caso, un aspecto que pudo haber influido en estos resultados es el escaso control de la variable objeto de estudio, pues para el reconocimiento de las expresiones faciales tienen que ver el estado emocional del observador, el feedback emocional, la imitación, la influencia del contexto, las diferencias individuales y las expectativas y atribuciones sobre el estado emocional (Fernández \& Sánchez, 2002); aspectos que no fueron controlados.

Por otro lado, existe evidencia de que la alexitimia se relaciona con la masculinidad y/o los roles tradicionales del sexo masculino (Garaigordobil, 2013). Algunos argumentos a favor de estos hallazgos se asocian con la lateralización del cerebro del varón, ya que en la mujer existe una comunicación interhemisférica más fluida, lo cual no sucede en los varones, por tanto, estos tendrían más desventajas para procesar información de carácter emocional. Otros datos relacionan la alexitimia con la agresividad, pues la primera se considera una variable motivadora de la agresión. En mujeres se ha encontrado, por ejemplo, que la alexitimia es un factor de 
riesgo de violencia sufrida (Moral de la Rubia \& Ramos-Basurto, 2015). Además, la dificultad para reconocer sentimientos puede favorecer estados afectivos disfóricos e ira (Rueda, Pérez-García, Sanjuán \& Ruíz, 2006), hecho que se ha tomado en cuenta como un factor explicativo de la conducta agresiva en niños y adolescentes (Arias, 2013).

Con respecto a la edad, se ha reportado que a mayor edad se tienen puntajes más altos de alexitimia, sobre todo a partir de los 55 años (Garaigordobil, 2013). Aunque el ejercicio físico suele disminuir las manifestaciones alexitímicas en los adultos mayores, esto no sucede en el deporte de tipo competitivo (Medina-Porqueres et al., 2016).

En la esfera social, se ha visto que la alexitimia es más frecuente en individuos de nivel socioeconómico bajo (Alonso-Fernández, 2011), y que, aunque se reconoce que existen diferencias culturales en la expresión de las emociones, se ha reportado que la alexitimia suele mantenerse estable en diversas culturas (Páez \& Casullo, 200o). En ese sentido, las emociones tienen diversos componentes que intervienen en la alexitimia, como son el cognitivo-subjetivo, fisiológico, comportamental y expresivo. Dado que la alexitimia implica déficit en el procesamiento emocional, el componente cognitivo tiene cierta relevancia. Por ejemplo, en Arequipa, Galagarza Pérez (2006) reportó que, en una muestra de 1023 adolescentes de la ciudad, el locus de control externo se asocia con mayor riesgo suicida.

A su vez, dados los déficit de la expresión emocional, la alexitimia se ha relacionado con trastornos del espectro autista (Hill, Berthoz \& Frith, 2004), pues tal y como reportó Leo Kanner (1987), los niños autistas tienen dificultades para expresar su emociones; aunque al principio se pensaba que esta reacción estaba mediada por la conducta fría y distante de las madres, luego se determinó que su etiología tenía un trasfondo neurológico.

Ahora bien, dentro de los rasgos secundarios de la alexitimia se han mencionado el conformismo social, la impulsividad, las relaciones interpersonales estereotipadas y la inmadurez (Moral de la Rubia \& Retamales, 2000). Sin embargo, algunos datos no escapan a contradicciones, porque las mujeres presentan mayor conformismo social que los varones (Arias \& Osorio, 2014), pero la alexitimia es más frecuente en los segundos. Por otro lado, existe evidencia de que la alexitimia puede explicarse como un rasgo de personalidad. Por ejemplo, Moral de la Rubia (2014) reportó que, al aplicar el MMPI y la Escala de Alexitimia de Toronto a 362 estudiantes universitarios, se encontraron tres factores de la personalidad que explican en su conjunto el $63.21 \%$ de la varianza de la alexitimia. Estos factores fueron el psicoticismo, la depresión y el factor somatomorfe. 
En resumen, la alexitimia es un desorden emocional, donde la persona que la tiene presenta dificultades para reconocer y expresar sus emociones o sentimientos, o los de otra persona, y se asocia a síntomas físicos y/o somáticos, que pueden abarcar depresión, ansiedad, consumo de sustancias psicoactivas, etc. Para fines de este presente estudio, se pretende valorar su grado de relación con la cohesión familiar. Al respecto, se ha reportado que la implicación de los padres para con los hijos se relaciona con el autoconcepto de los segundos, pero la cohesión familiar tiene poca relación con el autoconcepto y su rendimiento académico (González-Pienda et al., 2003).

En ese sentido, de acuerdo con el ciclo vital de la familia, cuando los hijos son pequeños se puede registrar un impacto mayor de la familia en ellos (Arias, 2013). Esto se demuestra en los estudios realizados en Arequipa sobre los efectos de la familia en la educación, donde, para el caso de los niños de primaria, se reportaron asociaciones entre la estructura familiar y el nivel de logro, de modo que las familias nucleares y extendidas se asociaron con un mayor nivel de logro de los niños (Arias, Quispe \& Ceballos, 2016); sin embargo, la satisfacción familiar no tuvo impacto en el rendimiento académico de los estudiantes universitarios (Laurie, Arias \& Ceballos, 2017).

Por otro lado, Alarcón (2014) ha reportado que, en una muestra de estudiantes universitarios de Lima, aquellos que provienen de familias más cohesionadas tienen mayores niveles de felicidad. No obstante, también se ha visto que los grupos familiares muy cohesionados pueden adquirir características alexitímicas (Alonso-Fernández, 2011). Otros estudios han reportado que la interacción con los padres se ha considerado como un factor protector de la alexitimia. De hecho, la disfuncionalidad parental predice las dificultades para la identificación de sentimientos, sobre todo el rechazo parental, que también se relaciona con ansiedad y consumo de sustancias psicoactivas, como la heroína (Torrado, Soares \& Bacelar-Nicolau, 2013).

En nuestro medio, empero, no existen estudios publicados sobre alexitimia, aunque sí los hay sobre inteligencia emocional, que podría considerarse como la antinomia de esta, pues se define como la capacidad de reconocer y gestionar las propias emociones y las de los demás, promoviendo relaciones interpersonales positivas y satisfactorias (Goleman, 1997). En el Perú, este año, se ha publicado el análisis psicométrico del Cuestionario de Alexitimia para Niños, en una muestra de 265 adolescentes peruanos de 11 a 18 años, con buenos indicadores de bondad de ajuste y validez convergente. Para ello, se aplicaron la prueba de alexitimia de Taylor, Bagby y Parker (2003), y una prueba de ajuste parental. Asimismo, la prueba presentó un mejor ajuste con dos factores: dificultad para describir sentimientos y dificultad para identificarlos. También se reportó que a mayor alextimia menor era el ajus- 
te parental (Yearwood, Vliegen, Luyten, Chau \& Corveleyn, 2017). Hace dos años, Chunga investigó las relaciones entre la alexitimia y los trastornos alimentarios en adolescentes femeninas en edad escolar, encontrando correlaciones moderadas y significativas entre ambas variables (Chunga, 2015).

Un modelo muy usado en nuestro país (véase Ferreira Rocha, 2003; Capa, Vallejos \& Cárdenas, 2010; Mayorga \& Ñiquén, 2010), para investigar la funcionalidad familiar, es el Modelo circumplejo de David H. Olson, que responde a una concepción sistémica de la familia (Arias, 2012; Haley, 2002; Minuchin \& Fishman, 1996; Ochoa, 2004). Este plantea tres dimensiones: cohesión, adaptabilidad y comunicación. El cruce de estas dimensiones genera 16 categorías de familias según el grado de su interacción familiar. La cohesión es el vínculo emocional que los miembros de la familia tienen entre sí, y viene dada por el tiempo que pasan juntos, las coaliciones, los espacios que ocupan y los límites familiares. La adaptabilidad tiene que ver con la medida en que el sistema familiar es flexible, y está determinada por el control, la disciplina, el estilo de negociación, los roles familiares y las reglas que rigen la familia. La comunicación facilita el movimiento en las dimensiones de cohesión y adaptabilidad (Olson, 1993).

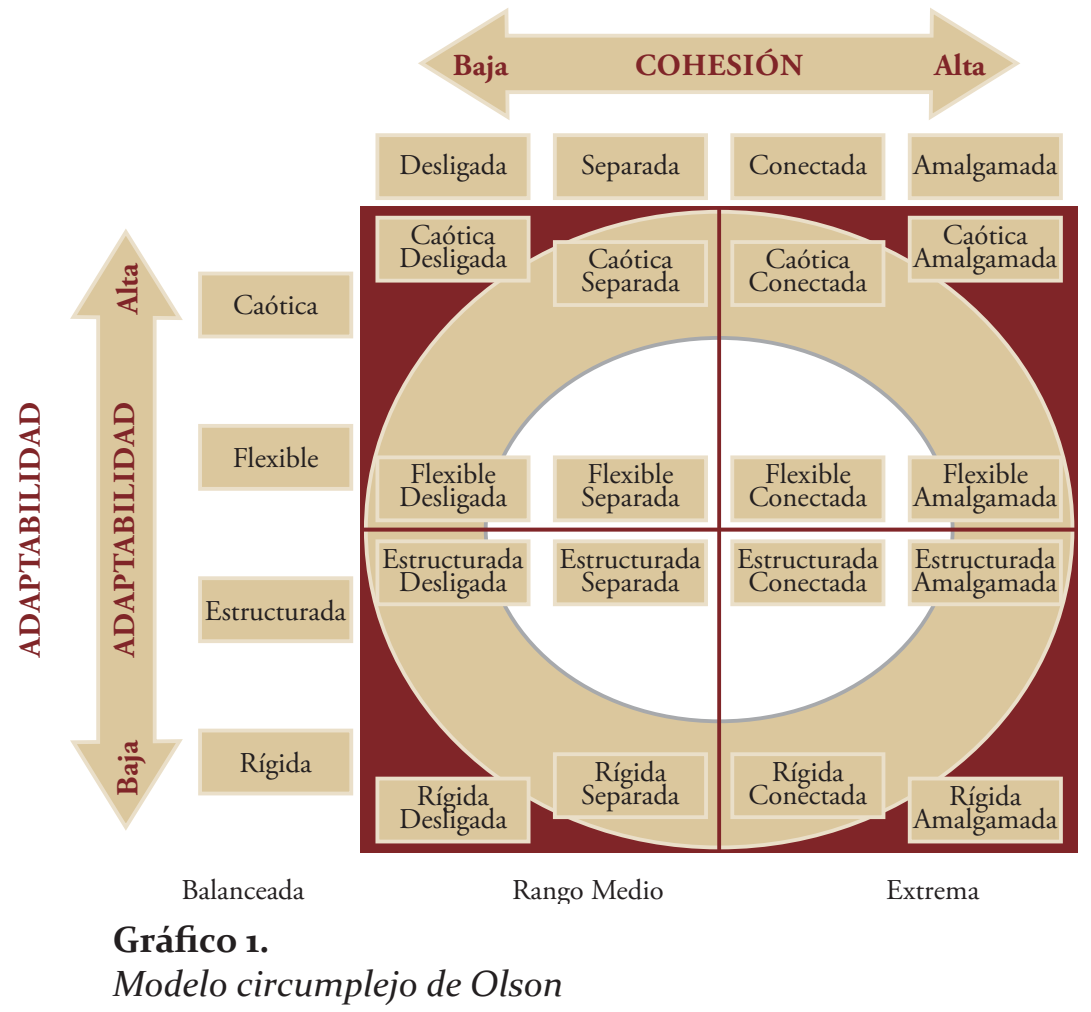


Asimismo, la cohesión determina cuatro niveles: desligada o no relacionada, separada o semirelacionada, conectada o relacionada y amalgamada o aglutinada; mientras que también la adaptabilidad determina cuatro niveles: caótica, flexible, estructurada y rígida. Una familia funcional se ubica en un rango balanceado cuando tiende a ser semirelacionada flexible, semirelacionada estructurada, relacionada flexible o relacionada estructurada. Es decir que los extremos, tanto bajos como altos, de cohesión y adaptabilidad son negativos (Olson, Portner \& Lavee, 1985).

Ahora bien, la presente investigación pretende llenar el vacío conceptual que existe en nuestro país sobre un tema tan importante como la alexitimia en relación con la dinámica familiar. Para ello, se formularon las siguientes preguntas de investigación: ¿Cuáles son los tipos de cohesión y adaptabilidad familiar predominantes en los estudiantes de ingeniería? ¿Cuál es la prevalencia de alexitimia entre los estudiantes de ingeniería que forman parte de la muestra? ¿Qué diferencias se pueden hallar en la alexitimia y la cohesión familiar, en función del sexo de los estudiantes?

\section{Metodología}

\section{Muestra}

La muestra está constituida por estudiantes universitarios del noveno semestre de la Facultad de Ingeniería de una universidad privada de la localidad. De manera que, de un total de 423 alumnos matriculados, la muestra quedó conformada por 319 estudiantes, siendo 184 varones $(57.7 \%)$ y 135 mujeres (42.3\%). La edad media fue de 21.9 años, con \pm 3.59 de desviación estándar. El método de selección de la muestra fue no probabilístico, por medio de la técnica de muestreo por cuotas.

\section{Instrumentos}

Se aplicó la versión en español del Cuestionario FACES III, en formato autoadministrado de 20 ítems, que se basa en el Modelo circumplejo de Olson. En estudios previos se han descrito las cualidades psicométricas del instrumento, con muestras de estudiantes peruanos, como el que desarrolla en Lima Bazo-Alvarez et al. (2016), con una muestra de 910 adolescentes. Su estudio reporta que la escala de cohesión tiene una confiabilidad moderadamente alta $(\Omega=0.85)$, lo mismo que la escala de adaptabilidad $(\Omega=0.86)$. La validez de constructo confirmó la bondad de ajuste del modelo de dos factores con diez ítems cada uno, mediante el análisis factorial confirmatorio que se practicó.

También se aplicó la Escala de Alexitimia de Toronto, que consta de 20 ítems y que fue creada por Taylor, Bagby y Parker en 1985. La prueba puntúa sus respuestas del 
1 a 5 en una escala tipo Likert, por lo que se puede obtener un puntaje dentro de un rango de entre 20 y 100, considerándose como «alexitímico» aquel que logra una puntuación mayor a 61 (Taylor, Bagby \& Parker, 2003). La escala presenta una estructura ortogonal tridimensional que explica el $31 \%$ de la varianza total. El Factor I comprende los ítems 1, 3, 6, 7, 9, 13 y 14, y se refiere a las dificultades para expresar emociones. El Factor II comprende los ítems 2, 4, 11, 12 y 17, y se refiere a las dificultades para identificar emociones. El Factor III comprende los ítems 5, 8, 10, 15, $16,18,19$ y 20 , y se refiere al pensamiento externamente orientado. La prueba tiene un índice de consistencia interna moderado-alto, obtenido por medio de la prueba Alfa de Cronbach $(\alpha=0.81)$.

\section{Procedimientos}

Como primer paso, se procedió a evaluar a los estudiantes de forma grupal, repartiéndoseles los cuestionarios del FACES III y dándoles las instrucciones correspondientes de la siguiente manera: «A continuación podrán observar una serie de afirmaciones, junto a estas podrán ver números del 1 al 5 , los que califican las respuestas correspondientes, siendo 1 Nunca y 5 Siempre. Por favor, respondan con la mayor sinceridad posible, no hay respuestas correctas ni incorrectas». Al terminar con el cuestionario, se les repartió el de Alexitimia (TAS-20) y se les dio las siguientes instrucciones: «Pueden observar que de igual forma que en el cuestionario anterior, lo que deben hacer es marcar el número que mejor los describa a ustedes en cuanto a las afirmaciones presentadas». Al terminar, se procedía a recoger ambos cuestionarios, para, seguidamente, realizar el procesamiento de la información mediante pruebas estadísticas no paramétricas.

\section{Resultados}

En primer lugar, se presentarán los datos descriptivos de la cohesión, adaptabilidad familiar y la alexitimia, para luego valorar las comparaciones según los sexos de los estudiantes. Así tenemos que, del $100 \%$ de personas evaluadas, el tipo de cohesión predominante es el semirelacionado, con un porcentaje de $43.26 \%$, seguido por el de tipo no relacionado, que comprende el $23.30 \%$. Asimismo, del $100 \%$ de personas evaluadas, el tipo de adaptabilidad predominante es el caótico, con un $47.02 \%$, seguido por el tipo flexible, con un $36.99 \%$. Con respecto a la alexitimia, del $100 \%$ de personas evaluadas (319 en total), un $67 \%$ de estas no presenta alexitimia (esto es, 214 personas), mientras que un $32 \%$ presenta algún grado de alexitimia, es decir, un total de 105 personas.

$\mathrm{Al}$ hacer comparaciones según el sexo de los estudiantes, el 26.67\% de mujeres $(\mathrm{N}=$ 36) presenta alexitimia, y el $73.3 \%$, no. A su vez, se aprecia que el $37.50 \%$ de varo- 
nes $(\mathrm{N}=69)$ presenta alexitimia y el $62.5 \%$ no la presenta. La diferencia encontrada fue estadísticamente significativa $(\mathrm{p}<0.050)$, tras aplicar la prueba Chi cuadrado $\left(\mathrm{x}^{2}=4.14 ; \mathrm{gl}=1 ; \mathrm{p}=0.041\right)$, por lo tanto, existe una proporción significativamente mayor de alexitimia en varones.

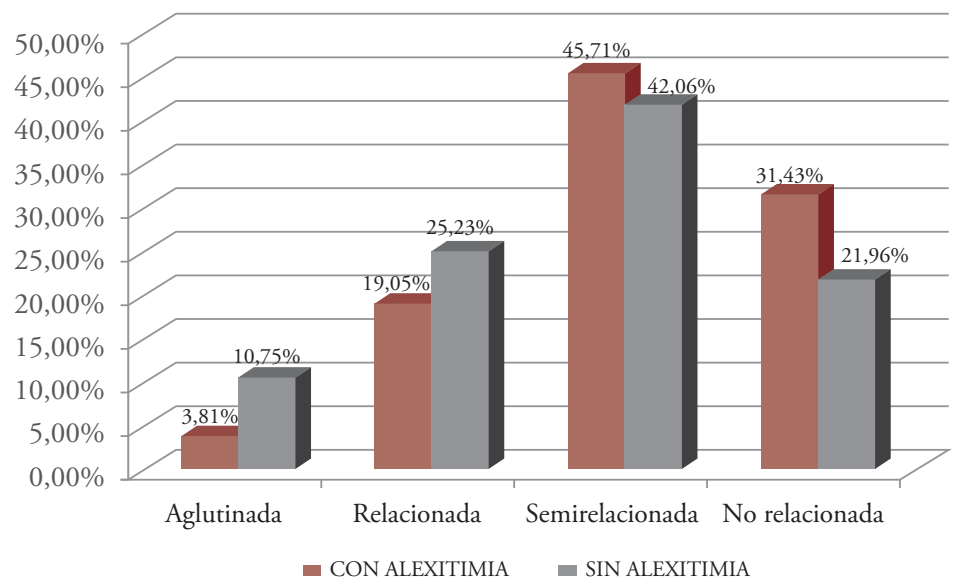

\section{Gráfico 2.}

Tipo de cohesión en personas con y sin alexitimia

En la Gráfica 2 podemos observar que, de las 105 personas con alexitimia, la mayoría (es decir, $45.7 \%$ ) presenta un tipo de cohesión semirelacionada, el 31.4\% tiene un tipo de cohesión no relacionada y el 19.0 \% y $3.8 \%$ tienen un tipo de cohesión familiar relacionada y aglutinada, respectivamente. Además, de las 214 personas sin alexitimia, la mayoría (esto es, $42.1 \%$ ) presenta un tipo de cohesión semirelacionada, el $25.2 \%$ tiene un tipo de cohesión relacionada y el $22.0 \%$ y $10.7 \%$ tienen un tipo de cohesión familiar no relacionada y aglutinada, respectivamente. Al aplicar la prueba estadística Chi cuadrado $\left(\mathrm{x}^{2}=7.90, \mathrm{gl}=3 ; \mathrm{p}=0.0481\right)$, se encontró una relación significativa entre las personas con alexitimia y un tipo de cohesión familiar semirelacionada o no relacionada, lo mismo sucedió entre personas sin alexitimia $\mathrm{y}$ un tipo de cohesión semirelacionada o relacionada $(\mathrm{p}<0.05)$. 


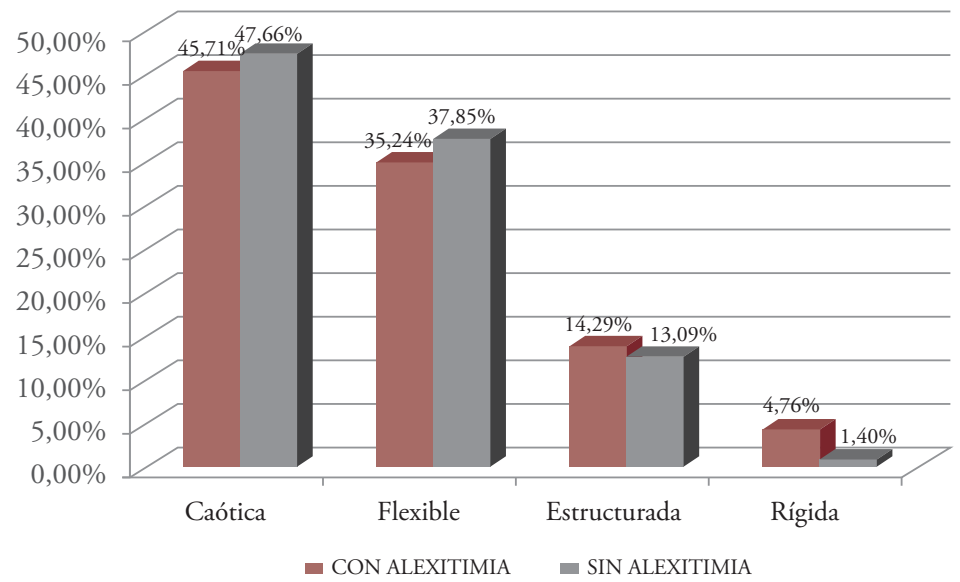

Gráfico 3.

Tipo de adaptabilidad en personas con y sin alexitimia

En la Gráfica 3 podemos observar que, de las 105 personas con alexitimia, la mayoría (esto es, $45.7 \%$ ) presenta un tipo de adaptabilidad caótica, el $35.2 \%$ tiene un tipo de adaptabilidad flexible y el $14.3 \%$ y $4.8 \%$ tienen un tipo de adaptabilidad familiar estructurada y rígida, respectivamente. También podemos observar que, de las 214 personas sin alexitimia, el $47.7 \%$ presenta un tipo de adaptabilidad caótica, el $37.9 \%$ tiene un tipo de adaptabilidad flexible y el $13.1 \%$ y $1.4 \%$ tienen un tipo de adaptabilidad familiar estructurada y rígida, respectivamente. Al aplicar la prueba Chi cuadrado, no se encontró diferencia significativa $\left(\mathrm{x}^{2}=3.43 ; \mathrm{gl}=3 ; \mathrm{p}=0.3295\right)$ en la adaptabilidad entre personas con y sin alexitimia.

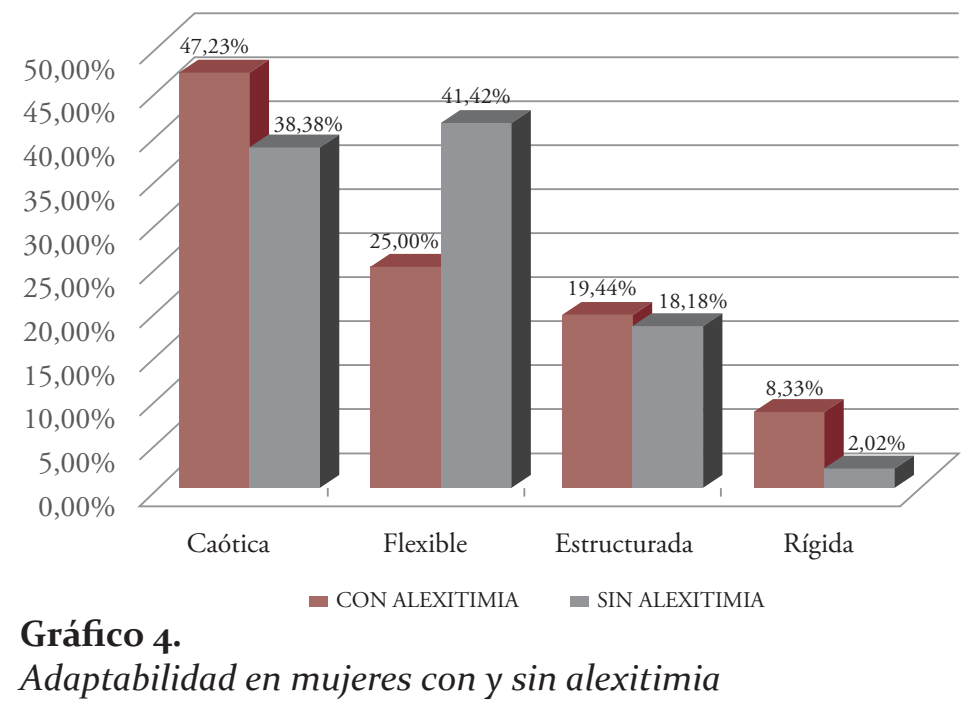


En la Gráfica 4 podemos observar que, del $100 \%(\mathrm{~N}=36)$ de mujeres que presenta alexitimia, el $47.23 \%$ tiene una adaptabilidad de tipo caótica, el $25 \%$, una adaptabilidad de tipo flexible, y el $19.44 \%$ y $8.33 \%$, una adaptabilidad de tipo estructurada y rígida, respectivamente. También podemos observar que, del $100 \%(\mathrm{~N}=99)$ de mujeres que no presenta alexitimia, el $41.42 \%$ tiene una adaptabilidad de tipo flexible, el $38.38 \%$, una adaptabilidad de tipo caótica, el $18.18 \%$, una adaptabilidad de tipo estructurada, y el $2.02 \%$ presenta una adaptabilidad de tipo rígida. No se encontraron diferencias significativas en la adaptabilidad de mujeres con y sin alexitimia, tras aplicar la prueba Chi cuadrado $\left(\mathrm{x}^{2}=5.29 ; \mathrm{gl}=3 ; \mathrm{p}=0.1517\right)$.

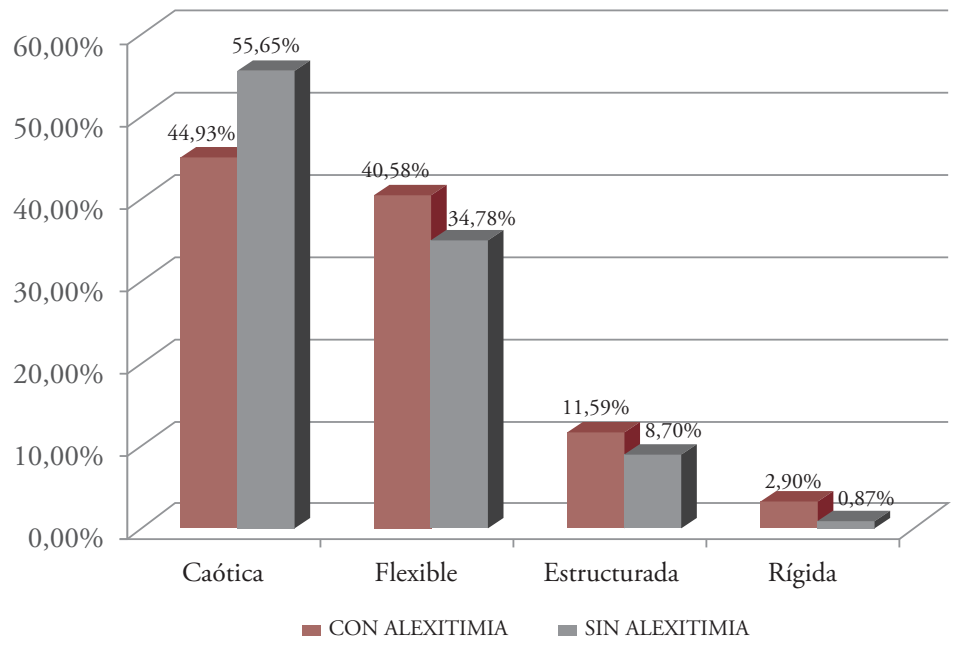

\section{Gráfico 5.}

Adaptabilidad en varones con y sin alexitimia

En el Gráfico 5 podemos observar que, del $100 \%(\mathrm{~N}=69)$ de varones que presenta alexitimia, el $44.93 \%$ tiene una adaptabilidad de tipo caótica, el $40.58 \%$, una adaptabilidad de tipo flexible, y el $11.59 \%$ y $2.90 \%$, una adaptabilidad de tipo estructurada y rígida, respectivamente. Del $100 \%(\mathrm{~N}=115)$ de hombres que no presenta alexitimia, el 55.65\% tiene una adaptabilidad de tipo caótica, el 34.78\%, una adaptabilidad de tipo flexible, y el $8.70 \%$ y $8.70 \%$, una adaptabilidad de tipo estructurada y rígida, respectivamente. Al aplicar la prueba Chi cuadrado, no se encontraron diferencias significativas $\left(\mathrm{x}^{2}=2.81 ; \mathrm{gl}=3 ; \mathrm{p}=0.4215\right)$ en la adaptabilidad familiar y la presencia de alexitimia en varones. 


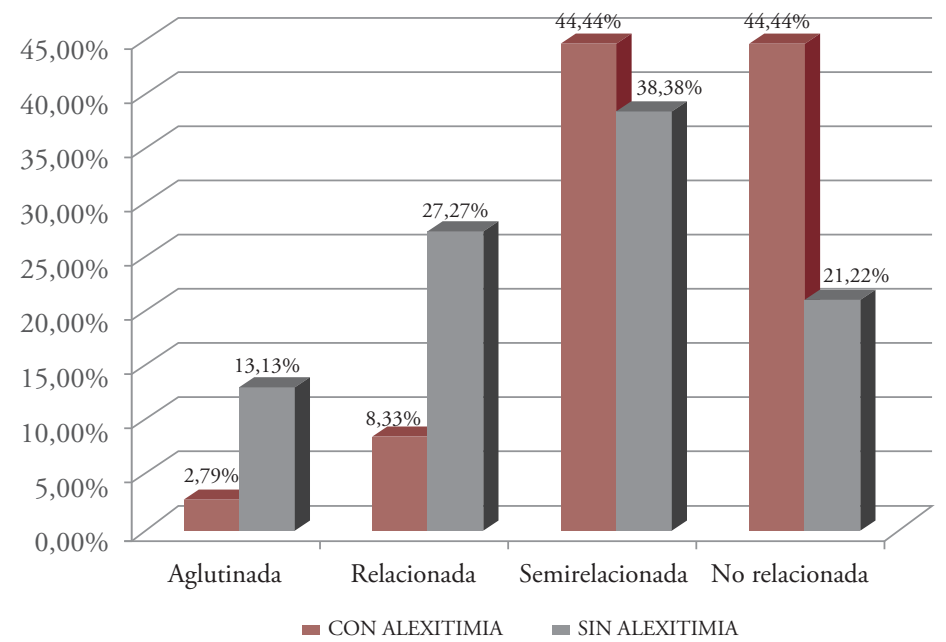

Gráfico 6.

Cohesión en mujeres con y sin alexitimia

En el Gráfico 6 podemos observar que, del $100 \%(\mathrm{~N}=36)$ de mujeres que presenta alexitimia, el $44.44 \%$ tiene tanto una cohesión de tipo no relacionada como semirelacionada, el $8.33 \%$, una cohesión de tipo relacionada, y el $2.78 \%$, una cohesión de tipo aglutinada. En el $100 \%(\mathrm{~N}=99)$ de mujeres que no presenta alexitimia, el $38.38 \%$ tiene una cohesión de tipo semirelacionada, el $27.27 \%$, una cohesión de tipo relacionada, el $21.22 \%$, una cohesión no relacionada. La diferencia de la cohesión familiar en mujeres con y sin alexitimia fue altamente significativa $\left(\mathrm{x}^{2}=12.43\right.$ $\mathrm{gl}=3 ; \mathrm{p}=0.0060$ ), predominando el tipo de cohesión no relacionada entre mujeres con alexitimia.

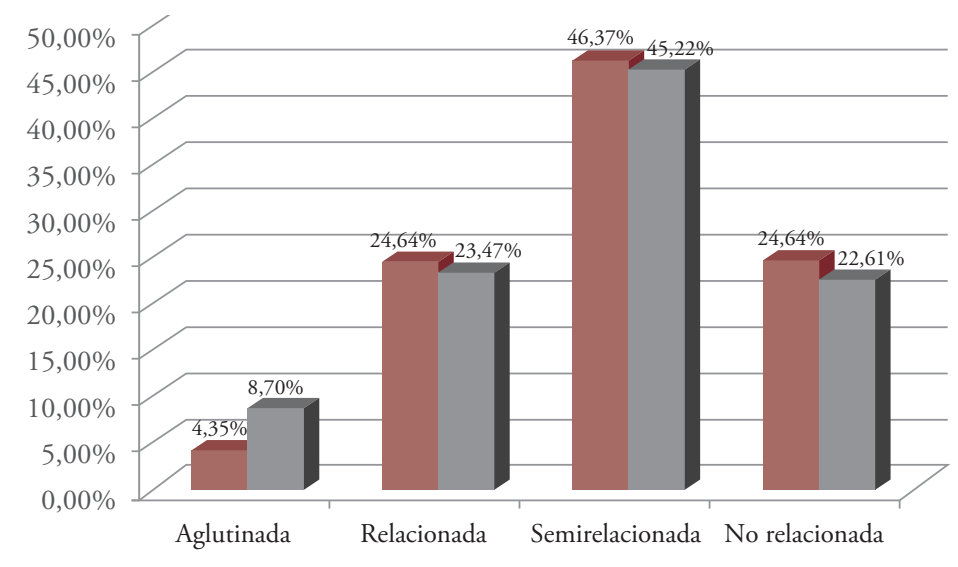

Gráfico 7 .

- CON ALEXITIMIA $\square$ SIN ALEXITIMIA

Cohesión en varones con y sin alexitimia 
En la Gráfica 7 podemos observar que, del $100 \%(\mathrm{~N}=69)$ de varones que presenta alexitimia, el $46.37 \%$ tiene una cohesión semirelacionada, el $24.64 \%$ tiene tanto una cohesión de tipo relacionada como no relacionada, y el $4.35 \%$, una cohesión de tipo aglutinada. Del $100 \%(\mathrm{~N}=115)$ de varones que no presenta alexitimia, el $45.22 \%$ tiene una cohesión de tipo semirelacionada, el $23.47 \%$, una cohesión de tipo relacionada, el $22.61 \%$ presenta una cohesión de tipo no relacionada, y el $8.70 \%$, una cohesión de tipo aglutinada. Al aplicar la prueba de comparación, no se encontró diferencia significativa $\left(\mathrm{x}^{2}=1.27 ; \mathrm{gl}=3 ; \mathrm{p}=0.737 \mathrm{O}\right)$ en la cohesión familiar en varones con y sin alexitimia.

\section{Discusión}

La alexitimia se caracteriza por la dificultad para expresar emociones verbalmente y tener una conciencia emocional precaria (Sivak \& Wiater, 1997). En ese sentido, si bien su núcleo patognomónico reside en la afectividad, la alexitimia se ha asociado a una gran variedad de síntomas y rasgos como: incapacidad de identificar, reconocer, nombrar o describir emociones o sentimientos propios o ajenos; pobreza de expresión verbal mímica o gestual; pensamiento concreto, pragmático y centrado en detalles externos; falta de capacidad introspectiva; reducción de la fantasía; psicomotilidad rígida; afrontamiento activo para resolver conflictos; discurso verbal monótono; dificultad para establecer contacto afectivo; relaciones interpersonales rígidas, inclinación al aislamiento; personalidad adusta, seria y aburrida (Alonso-Fernández, 2011).

Por ello, se considera que la alexitimia es agente causal de múltiples desórdenes psicológicos, como trastornos psicofisiológicos, trastornos de sueño, ansiedad, depresión, esquizofrenia, autismo, conductas agresivas, violencia intrafamiliar, etc. (Taylor, 1984). Sin embargo, a pesar de su importancia, la alexitimia ha sido poco investigada en nuestra sociedad y en nuestra ciudad, tanto es así que solo se cuenta con unos pocos estudios recientemente publicados (Yearwood et al., 2017; Chunga, 2015) y algunas tesis de pregrado.

En esta investigación se evaluó a estudiantes del noveno semestre de la Facultad de Ingeniería de una universidad privada local, con el objetivo de determinar el tipo de dinámica familiar, en función de la cohesión y la adaptabilidad, y la presencia de alexitimia. Se realizaron comparaciones de ambas variables, en función del sexo, y se encontraron los resultados que se detallan a continuación.

Se reportó la presencia de alexitimia en el $32.92 \%$ de la muestra evaluada. El tipo de cohesión familiar predominante en personas con alexitimia fue semirelacionado en 
el $43.26 \%$ de los casos, mientras que el tipo de adaptabilidad familiar predominante en personas con alexitimia es el caótico, con un $47 \%$ de incidencia. Por otro lado, no se encontró diferencias significativas en la adaptabilidad familiar entre personas con y sin alexitimia, lo que nos sugiere que la adaptabilidad no es un factor influyente en la presencia de alexitimia. Sin embargo, se pudo verificar que la alexitimia está presente en una proporción significativamente mayor de estudiantes varones que en mujeres, lo que corrobora los reportes previos acerca de su prevalencia en personas de sexo masculino (Alonso-Fernández, 2011; Garaigordobil, 2013; Sivak \& Wiater, 1997; Moral de la Rubia \& Ramos-Basurto, 2015).

Asimismo, la asociación entre alexitimia y cohesión familiar fue altamente significativa en el sexo femenino, pero en aquellas mujeres que presentan una cohesión familiar de tipo no relacionada o semirelacionada. Este dato contradice lo dicho por Alonso-Fernández (2011), cuando señala que las familias más cohesionadas son generadoras de alexitimia. Desde el punto de vista de Bowen (1998), si bien la familia es un núcleo básico de convivencia social, es fundamental la individuación de cada uno de sus miembros, por lo que una cohesión extrema, como es el caso de una familia aglutinada, podría ser perniciosa. Por otro lado, el modelo circumplejo (Olson, 1993) señala que la cohesión moderada, esto es, de tipo relacionada y semirelacionada, es saludable para los integrantes de la familia, mientras que los extremos, que incluyen los tipos no relacionado o aglutinado, no lo son.

Estos resultados nos hacen pensar en la posibilidad de que si se mejora la dinámica familiar, podría bajar la incidencia de alexitimia, lo cual tendría implicancias muy positivas para la salud mental de los padres y los hijos que conforman la familia. En ese sentido, la terapia más adecuada para la alexitimia es la psicoterapia breve, la psicoterapia interpersonal o el análisis introspectivo, dadas las características de las personas alexitímicas (Krystal, 1979; Fernández \& Yanoz, 1994). Sin embargo, una manera de prevenirla es promoviendo la expresión de las emociones en los niños, particularmente en los varones, que es a quienes más se les inhibe desde que son pequeños, y podría ser que, por ello, son quienes presentan mayor nivel de alexitimia.

Goleman (1997), por ejemplo, recomienda que, en la escuela, en lugar de decir presente cuando se llama lista, los estudiantes deberían comunicar su estado de ánimo, y hacer de esto una costumbre. Actividades como esta son fundamentales para promover una libre y adecuada expresión de las emociones; aunque en un nivel de educación superior se podrían emplear otras estrategias más acordes con la edad de los estudiantes, como son los trabajos grupales, los talleres de teatro y artes plásticas, tutorías personalizadas y orientación psicopedagógica. 
El presente estudio toca un tema poco atendido en nuestra comunidad, tanto a nivel local como nacional, por lo que revista de particular importancia continuar profundizando en las manifestaciones y relaciones de la alexitimia con otras variables. Sin embargo, nuestro estudio cuenta con algunas limitaciones, entre las que podemos mencionar el escaso tamaño de la muestra y las limitaciones propias del procesamiento estadístico con pruebas no paramétricas.

Investigaciones futuras deben superar estas limitaciones y desarrollar nuevos estudios sobre la alexitimia en diversos contextos, como el clínico, social, educativo, organizacional, etc., donde no existen antecedentes investigativos en nuestro país. Por otra parte, estudios de corte psicométrico deberían ocuparse de validar y estandarizar los instrumentos utilizados para evaluar esta variable.

\section{Referencias}

Alarcón, R. (2014). Funcionamiento familiar y sus relaciones con la felicidad. Revista Peruana de Psicología y Trabajo Social, 3(1), 61-74.

Almeida, V., \& Machado, P. P. P. (2004). Somatização e alexitimia: Um estudo nos cuidados de saúde primarios. International Journal of Clinical and Health Psychology, 4(2), 285-298.

Alonso-Fernández, F. (2011). La alexitimia y su trascendencia clínica y social. Salud Mental, 34, 481-490.

Arias, W. L. (2012). Algunas consideraciones sobre la familia y la crianza desde un enfoque sistémico. Revista de Psicología de Arequipa, 2(1), 32-46.

Arias, W. L. (2013). Agresión y violencia en la adolescencia: La importancia de la familia. Avances en Psicología, 21(1), 23-34.

Arias, W. L., \& Osorio, J. F. (2014). Estudio experimental del conformismo ante situaciones no estructuradas en estudiantes universitarios. Revista Peruana de Psicología y Trabajo Social, 3(2), 15-28.

Arias, W. L., Quispe, A. C., \& Ceballos, K. D. (2016). Estructura familiar y nivel de logro en niños y niñas de escuelas públicas de Arequipa. Perspectiva de Familia, $1(1)$, 35-62.

Bazo-Alvarez, J. C., Bazo-Alvarez, O. A., Aguila, J., Peralta, F., Mormontoy, W., \& Bennett, I. M. (2016). Propiedades psicométricas de la Escala de funcionalidad familiar FACES-III: Un estudio en adolescentes peruanos. Revista Peruana de Medicina Experimental y Salud Pública, 33(3), 462-470.

Bowen, M. (1998). De la familia al individuo. Barcelona: Paidós.

Capa, W., Vallejos, M., \& Cárdenas, R. (2010). Factores psicosociales y demográficos asociados al consumo de drogas en adolescentes de una zona urbano popular de Lima Metropolitana. Revista de Investigaciones Psicológicas, 1, 21-37. 
Chunga, L. (2015). Trastornos de la conducta alimentaria y alexitimia en adolescentes femeninas escolares. Revista de Psicología de la UCV, 17(2), 203-220.

Craparo, G., Gori, A., Dell'Aera, S., Costanzo, G., Fasciano, S., Tomasello, A., \& Vicario, C. M. (2016). Impaired emotion recognition is linked to alexithymia in heroin addicts. Peer Journal, 4, 201-212.

Diaz, S. (2011). Alexitimia y bienestar psicológico en pacientes con diagnóstico de trastorno gastrointestinal funcional. III Congreso Internacional de Investigación y Práctica Profesional en Psicología. Universidad de Buenos Aires, Argentina.

Donges, U. S., Kersting, A., \& Suslow, T. (2014). Alesithymia and perception of emotional information: A review of experimental psychological findings. Universitas Psychologica, 13(2), 745-756.

Fernández, J., \& Yanoz, S. (1994). Alexitimia: Concepto, evaluación y tratamiento. Psicothema, 6(3), 357-366.

Fernández, S., \& Sánchez, A. (2002). Deterioro de habilidades emocionales en la alexitimia: Implicaciones educativas. Papeles Salmantinos de Educación, 1, 151-181.

Ferreira Rocha, A. M. (2003). Sistema de interacción familiar asociado a la autoestima de menores en situación de abandono moral y prostitución. Revista de Investigación en Psicología, 16(2), 58-80.

Galagarza Pérez, L. (2006). Locus de control y riesgo suicida en adolescentes. Revista de Psicología de la UCSM, 3, 37-43.

Garaigordobil, M. (2013). Sexism and alexithymia: Correlations and differences as a function of gender, age and educational level. Anales de Psicología, 29(2), 368-377.

García, V. R., Rodríguez, S. E., Córdova, A. J., \& Fernández-Cáceres, C. (2016). Alexitimia y consumo de drogas en hombres y mujeres en tratamiento. Revista Internacional de Investigación en Adicciones, 2(2), 13-21.

Goleman, D. (1997). Inteligencia emocional. Barcelona: Kairós.

González-Pienda, J. A., Núñez, J. C., Álvarez, L., Roces, C., González-Pumariega, S., González, P., Muñiz, R., Valle, A., Cabanach, R. G., Rodríguez, S., \& Bernardo, A. (2003). Adaptabilidad y cohesión familiar, implicación parental en conductas autorregulatorias, autoconcepto del estudiante y rendimiento académico. Psicothema, 15(3), 471-477.

Haley, J. (2002). Terapia para resolver problemas. Nuevas estrategias para una terapia familiar eficaz. Buenos Aires: Amorrotu Editores.

Hill, E., Berthoz, S., \& Frith, U. (2004). Brief report: cognitive processing of own emotions in individuals with autistic spectrum disorder and in their relatives. Journal of Autism and Developmental Disorders, 34(2), 229-235. 
Kanner, L. (1987). Psiquiatría infantil. México: Siglo XXI.

Krystal, H. (1979). Alexithymia and Psychotherapy. American Journal Psychotherapy, 33, 17-31.

Krystal, H. (1988). Integration and Self-Healing: Affect, trauma, alexithymia. Hillsdate, N. J.: Analytic Press.

Laquatra, T. A., \& Clopton, J. R. (1994). Characteristics of alexithymia and eating disorders in college women. Addiction E Behavior, 19(4), 373-380.

Laurie, P., Arias, W. L., \& Ceballos, K. D. (2017). Satisfacción familiar y malestar psicológico como predictores del rendimiento académico en estudiantes universitarios [artículo sometido a revisión].

Ledux, L. (1999). El cerebro emocional. Buenos Aires: Editorial Planeta.

Martínez, F., \& Marín, J. (1997). Influencia del nivel de alexitimia en el procesamiento de estímulos emocionales en una tarea stroop. Psicothema, 9(3), 519-527.

Martínez, F., Montero, J. M., \& Cerra, J. de la (2002). Sesgos cognitivos en el reconocimiento de expresiones emocionales de voz sintética en la alexitimia. Psicothema, 14(2), 344-349.

Martínez, F., Ortiz-Soria, B., \& Ato-García, M. (2001). Subjective and autonomic stress responses in alexithymia. Psicothema, 13(1), 57-62.

Mayer, J., Di Paolo, M., \& Salovey, P. (1990). Perceiving affective content in ambiguous visual stimuli: a component of emotional intelligence. Journal of Personality Assessment, 54(3-4), 772-781.

Mayorga, E., \& Ñiquen, M. (2010). Satisfacción familiar y expresión de la cólera-hostilidad en adolescentes escolares que presentan conductas antisociales. Revista de Investigaciones Psicológicas, 1(1), 87-92.

Medina-Porqueres, I., Romero-Galisteo, R. P., Gálvez-Ruiz, P., Moreno-Morales, N., Sánchez-Guerrero, E., Cuesta-Vargas, J. S., Mate-Pacheco, F., \& Barón-López, F. J. (2016). Alexitimia y depresión en mayores que practican actividad física dirigida. Revista Iberoamericana de Ciencias de la Actividad Física y el Deporte, 5(3), 36-48.

Minuchin, S., \& Fishman, H. (1996). Técnicas de terapia familiar. México: Paidós.

Moral de la Rubia, J. (2014). Predicción de la alexitimia por escalas clínicas y de validación del MMPI. International Journal of Good Conscience, 9(1), 65-82.

Moral de la Rubia, J., \& Ramos-Basurto, S. (2015). Alexitimia como predictor directo y mediado por la depresión en la violencia de pareja. Revista Costarricense de Psicología, 34(1), 15-41.

Moral de la Rubia, J., \& Retamales, R. (2000). Sobre la alexitimia primaria y secundaria: Datos para la polémica. Psiquis, 22(4), 221-227. 
Morey, A. (2007). Género y habilidad para identificar estados emocionales en expresiones faciales o gestos. Revista de Psicología de la UCSM, 4, 64-73.

Ochoa, I. (2004). Enfoques de terapia familiar. Barcelona: Herder.

Olson, D. H. (1993). Circumplex model of family systems: Assessing functioning. En Walsh, F. (Ed.). Normal family processes (pp. 73-103). Nueva York: Guilford.

Olson, D. H., Portner, J., \& Lavee, Y. (1985). FACES III. St. Paul: University of Minnesota, Department of Family Science.

Páez, D., \& Casullo, M. M. (Eds.). (200o). Cultura y alexitimia. Buenos Aires: Paidós.

Roco, G., Baldi, G., \& Álvarez, G. (2014). Afrontamiento y alexitimia en una muestra de mujeres víctimas de violencia familiar en San Luis, Argentina. Alternativas en Psicología, 17 (29), 32-43.

Rueda, B., Pérez-García, A. M., Sanjuán, P., \& Ruíz, M. A. (2006). Relación entre alexitimia y hostilidad: ¿Una asociación influida por el malestar psicológico? Análisis y Modificación de Conducta, 32(143), 263-286.

Sifneos, P. E. (1972). Short-term psychotherapy and emotional crisis. Cambridge: Harvard University Press.

Sifneos, P. E. (1973). Prevalence of alexithymic characteristics in psychosomatic patients. Psychotherapy and psychosomatics, 22, 255-262.

Sivak, R., \& Wiater, A. (1997). Alexitimia, la dificultad para verbalizar afectos. Buenos Aires: Paidós.

Taylor, G. J. (1984). Alexithymia: concept, measurement and implications for treatment. American Journal of Psychiatry, 141, 725-732.

Taylor, G. J., Bagby, R. M., \& Parker, J. D. (2003). The 20-Item Toronto Alexithymia Scale: IV Reliability and factorial validity in different languages and cultures. Journal of Psychosomatic Research, 55(3), 277-283.

Taylor, G. J., Parker, J. D., Bagby, R. M., \& Bourke, M. P. (1996). Relationship between alexithymia and psychological characteristics associated with eating disorders. Journal of Psychosomatic Research, 41(6), 561-568.

Torrado, M. V., Soares, S., \& Bacelar-Nicolau, L. (2013). Alexithymia, emotional awareness and perceived dysfunctional parental behaviors in heroin dependents. International Journal of Mental Health $\mathcal{E}$ Addiction, 11(6), 703-718.

Torres-Hernández, M., López-García, S., Pedroza-Escobar, D., \& Escamilla-Tilch, M. (2015). Revista Médica del Instituto Mexicano del Seguro Social, 53(3), 268-272.

Yearwood, K., Vliegen, N., Luyten, P., Chau, C., \& Corveleyn, J. (2017). Psychometric properties of the Alexithymia Questionnaire for Children in a Peruvian simple of adolescents. Revista de Psicología de la PUCP, 35(1), 97-124. 